\title{
Research Paper \\ Different Perceptions of Time Passage among Older People: A Comparative Study in Terms of Age and Gender
}

\author{
Mohammad Ali Nazari ${ }^{1}$, *Sedigheh Forghani Bonab ${ }^{1}$, Jalil Babapour Kheireddin ${ }^{1}$
}

1. Department of Psychology, Faculty of Psychology \& Educational Sciences, University of Tabriz, Tabriz, Iran.

Citation: Nazari MA, Forghani Bonab S, Babapour Kheireddin J. [Different perception of time passage among older people: A comparative study in terms of age and gender (Persian)]. Iranian Journal of Ageing. 2016; 11(4):80-89. http://dx.doi.org/10.21859/sija-110180

http://dx.doi.org/10.21859/sija-110180

Received: 02 Oct. 2015 Accepted: 18 Jan. 2016

\section{ABSTRACT}

Objectives The perception of time is a fundamental and complicated cognitive skill, which allows us to perceive a series of events and activities and anticipate some events in the future. The passage of time is influenced by different contributions of the brain regions and basic cognitive processes, especially attention and memory. With regard to changes in the mentioned functions in different ages, this research aimed to determine the differences in time perception between young and older people.

Methods \& Materials This causal-comparative study included young (20-30 years) and elderly (60-70 years) women and men living in Tabriz and Marand cities, Iran. The study samples were recruited via convenient sampling method and comprised 64 individuals who were assigned to 2 groups: young ( $n=30 ; 15$ males and 15 females) and elderly subjects ( $n=27 ; 12$ males and 15 females). The measurements included mini-mental state examination test, Beck depression inventory, and time reproduction task. At first, the time reproduction task was individually performed for each subject. To prevent fatigue and its impact on time perception, Beck depression inventory and mini-mental state examination test were performed after the time reproduction task. In time reproductiontask, a circle was randomly presented in standard durations of 400,500 , and $600 \mathrm{~ms}$, as short time, andstandard durations of 1800,2000 , and $2200 \mathrm{~ms}$, as long time, successively. In total, 80 trials (40 trials for short times and 40 attempts for long times) were randomly and successively presented. Subjects should hold downthe space key to reproduce similar duration to the stimulus that had been previously presented. The data of all the subjects were analyzed by multivariate analyses test.

Results Our findings showed significant differences between 2 age groups (young and old) with regard to perception variable $(\mathrm{P}<0.007)$ in such a way that the older people had shorter reproduction in both short and long time intervals. However, the young people had longer reproduction in short time intervals and shorter reproduction (the time interval of motive existence) in long time intervals. With regard to gender, no significant difference was found between the women and men $(P<0.244)$.

Conclusion Research findings indicate that age affects time perception so that perceived time in the elderly people is shorter than the real physical time. Our findings also showed that if sources pay less attention to the passage of time or the memory capacity declines, the time estimation will be less. Based on cognitive changes in old age, the cognitive abilities of older people were found to decrease with increasing age. As the accurate processing of time intervals relates to memory capacity and attention, the changes in any cognitive process could affect the differences in the perception of time. Probably due to these factors, the reproduction of time in the older people shortens.

\section{Key words: \\ Gender, Age, Time} perception, Elderly

\section{* Corresponding Author:}

Sedigheh Forghani Bonab, MSc.

Address: Department of Psychology, Faculty of Psychology \& Educational Sciences, University of Tabriz, Tabriz, Iran.

Tel: +98 (937) 2831323

E-mail: sedighehforghani@yahoo.com 


\title{
آيا افراد كهنسال از تخذر زمان ادراك متفاوتى دارند؟: يك مطالعه مقايسهاى از نظر سن و جنس
}

\author{
محمدعلى نظرى'، "صديقه فرقانىبناب'، جليل بابايورخيرالدين' \\ ا - كروه روانشئاسي، دانشكده علوم تربيتى و روانشئاسى، دائشكاه تبريز، تبريز، ايران.
}

\begin{abstract}
Q

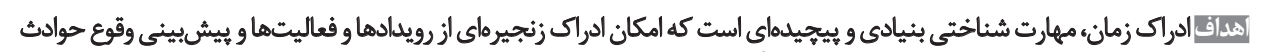

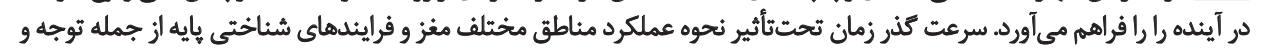

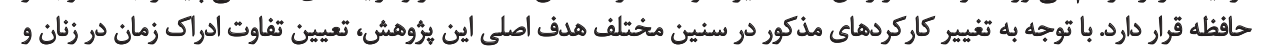
مردان جوان و كهئسال بود بوان.

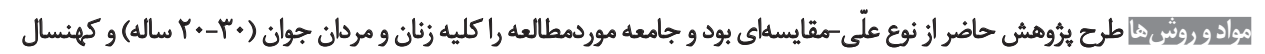

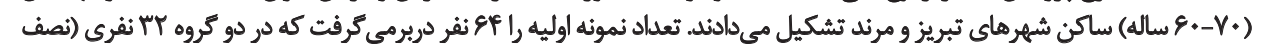

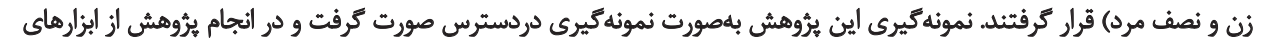

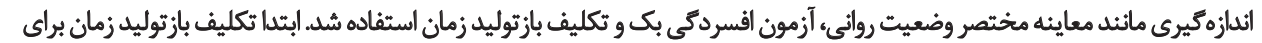

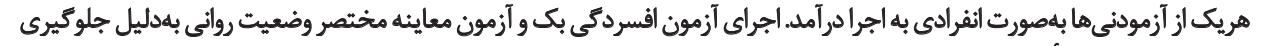

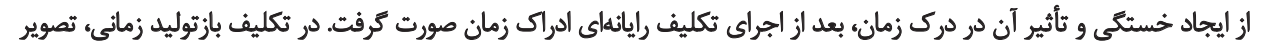

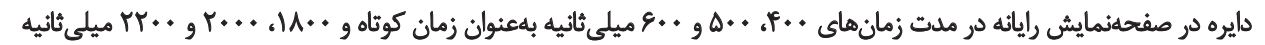

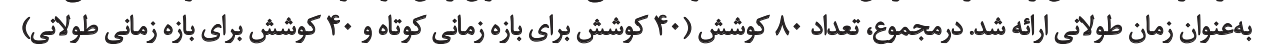

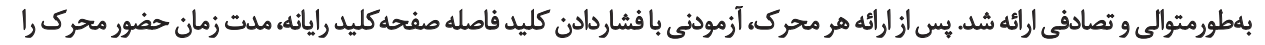

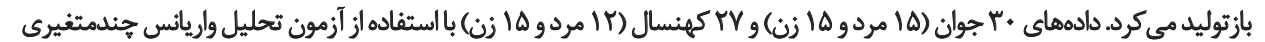

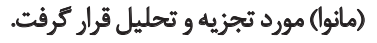

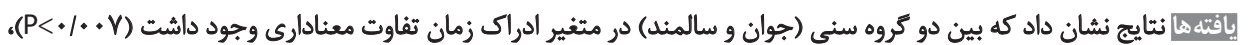

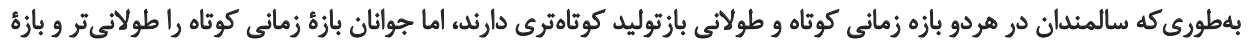

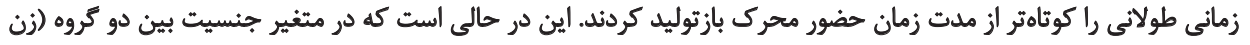

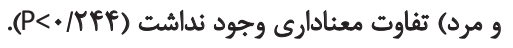

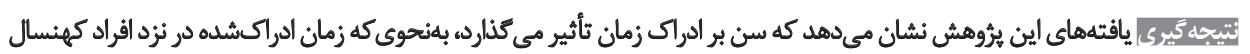

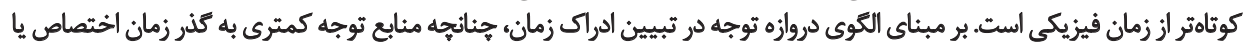

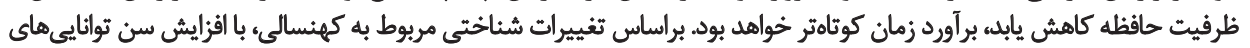

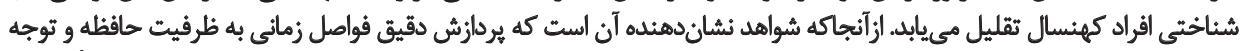

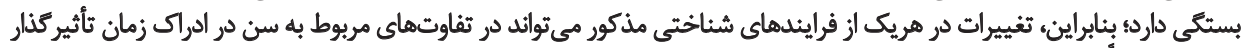

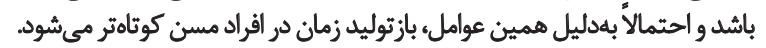

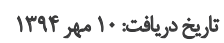

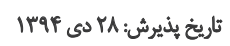

از نظر بسيارى از محققان، زمانبندى' يك مفهوم بنيادى در

از آغاز تفكر بشرى تابهامروز، ماهيت و جيستى زمان بلعنوان

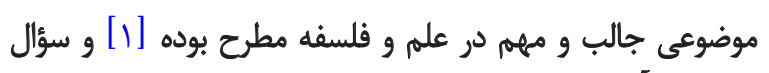

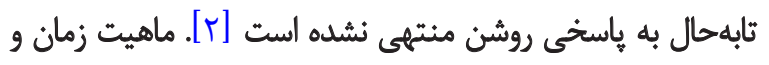




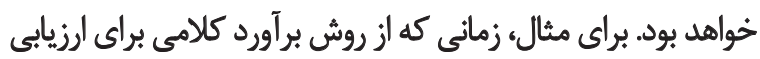

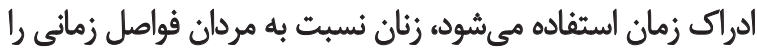

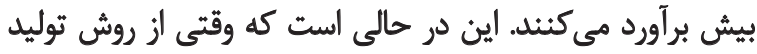

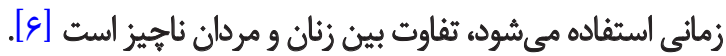

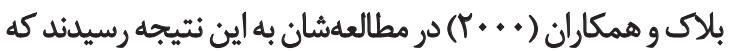

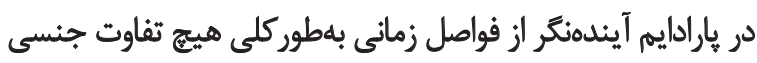

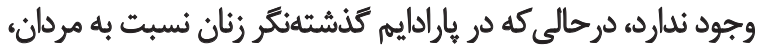

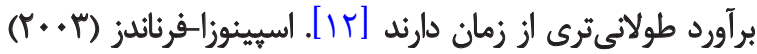

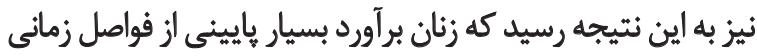
طولانى مثلاً در حدود ه دقيقه دارند [ه]].

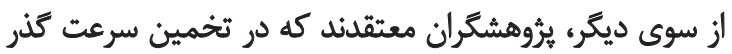

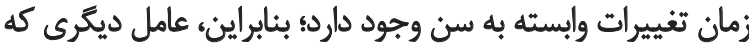

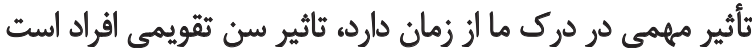

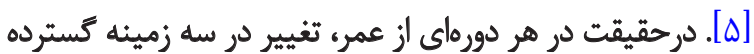

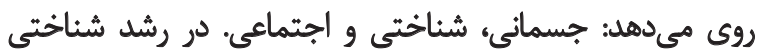

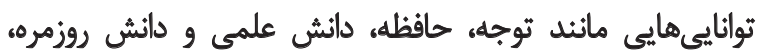

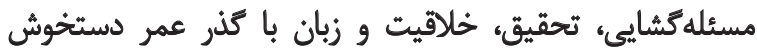

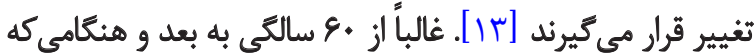

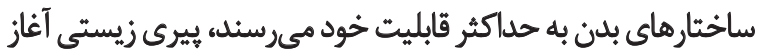

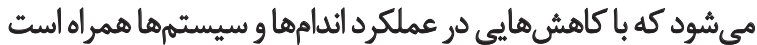
كه در تمام اعضاى كونهائا عموميت دارند

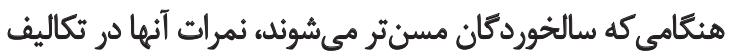

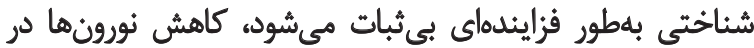

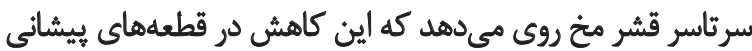

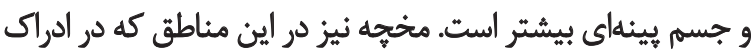

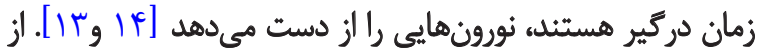

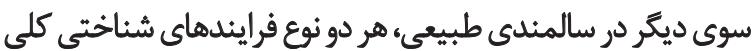

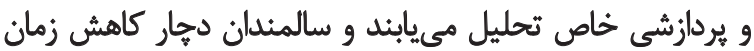

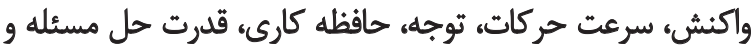

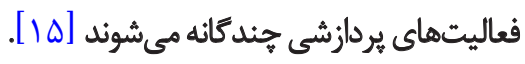

شواهد نشاندهنده آن است كه رشد توانايىهاى لازم جهت

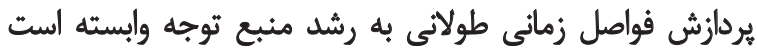

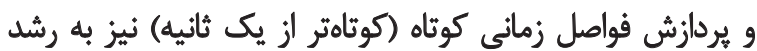

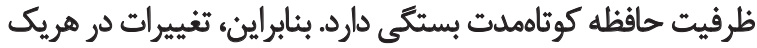

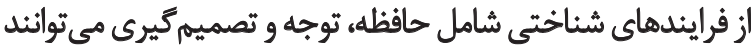

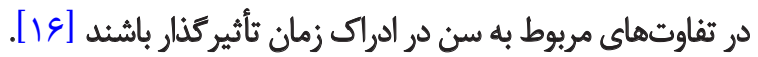
بسيارى از جنبههاى رفتارى و شناختى ما نيز با بردازش زمانى

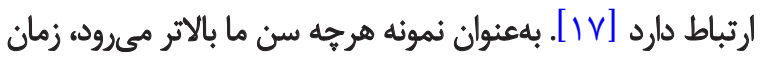

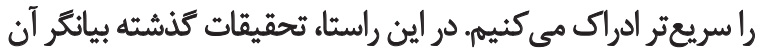

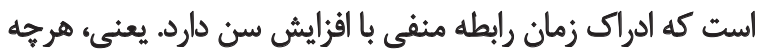

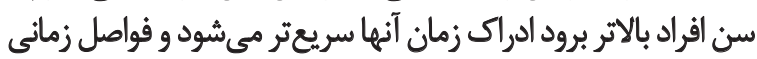

روانشناسى هحسوب مى فود و عملكرد كار آمد تا حدى به به توانايى

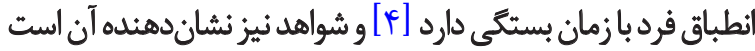

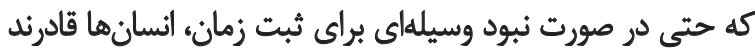

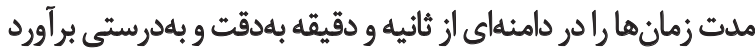

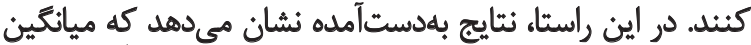
تخمينهاي ذهنى افراد با زمان واقعى برآوردشده تقريباً برابر است

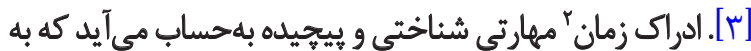

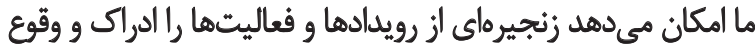

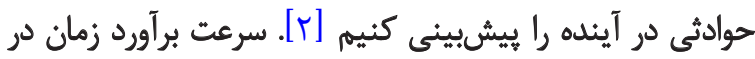

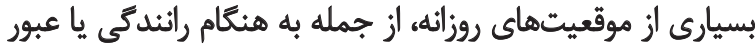

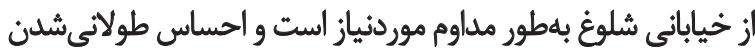

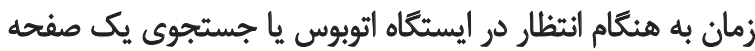

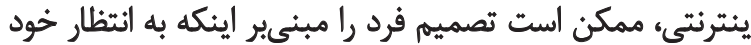

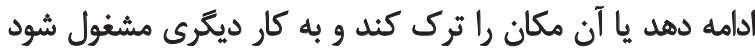

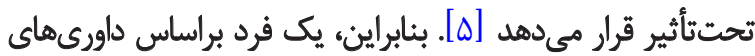

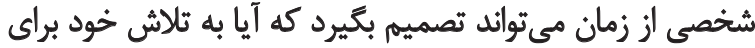

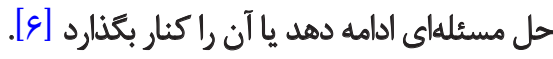

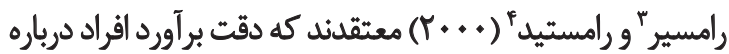

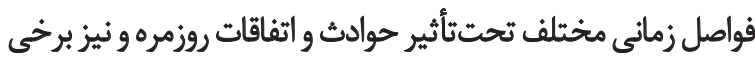

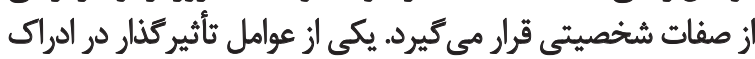

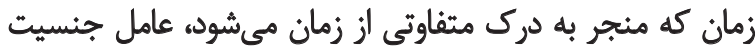

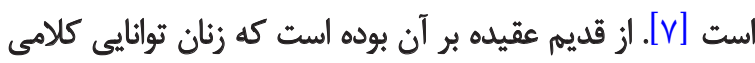

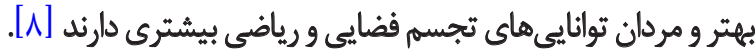

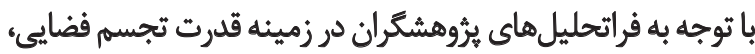

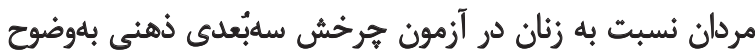

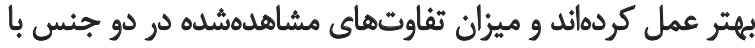

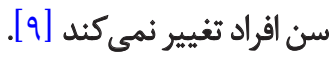
يروهشكران معتقدند كه فضا و زمان دو روى يك سكهاند و تفاوت

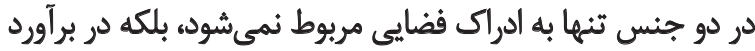

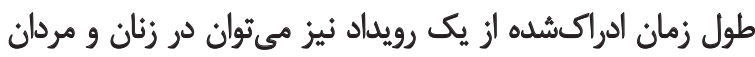

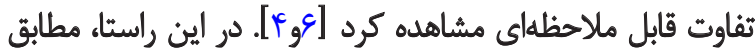

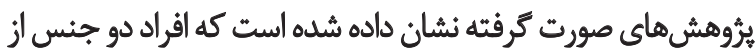

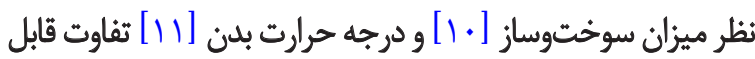

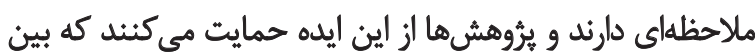

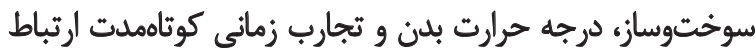

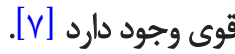
بررسى هاى فراتحليلى اخير نشان داده است كه تأثير جنسيت در ادراك زمان بسته به نوع روش و وريكرد بهان كاربردهشده متفاوت

2. Time perception

3. Rammsayer

4. Rammstedt 
بينايى؛ \&-عدم مصرف داروهاي روان كردان.

تعداد نمونه اوليه كه بهصورت دردسترس انتخاب شده بودند

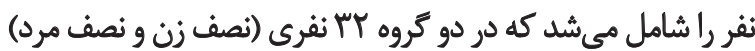

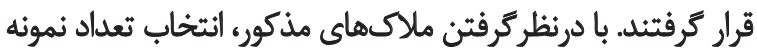

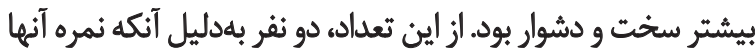

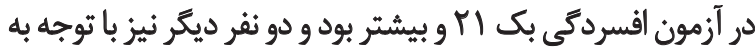

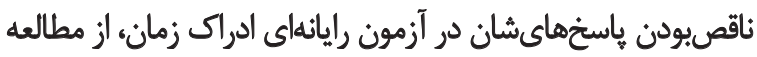

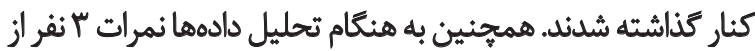

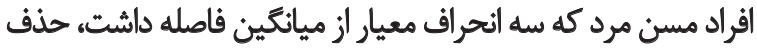

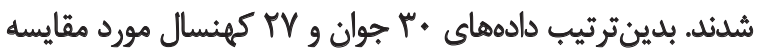

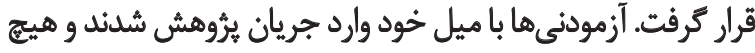

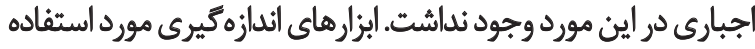

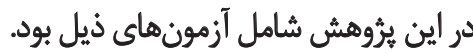

ازآنجاكه شواهد نشاندهئده آن است كه اختالالتى همجيون

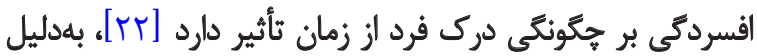

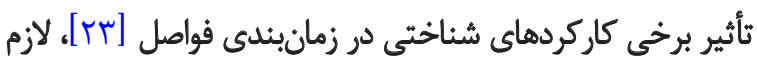

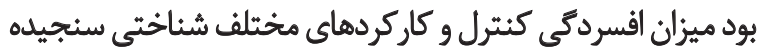

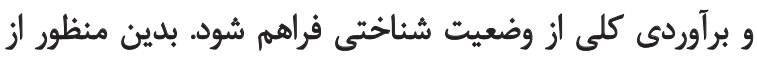

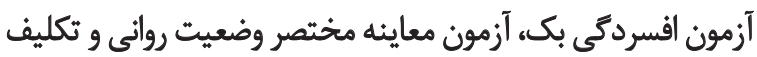

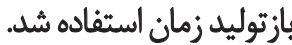

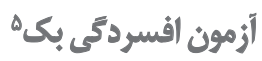

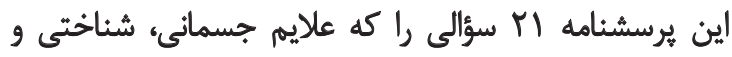

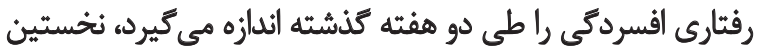

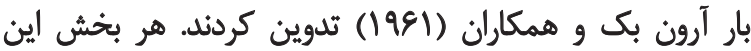

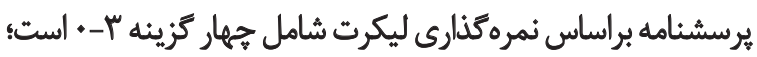

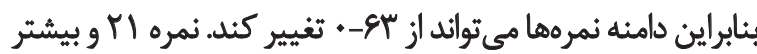

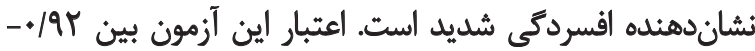

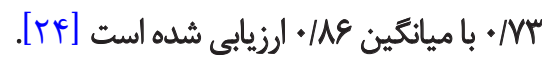

أزمون معايثه مختصر وضعيث روائى (MMSE)'

اين ابزار متداولترين ابزار غربال گرايى شناختى در سطح جهان

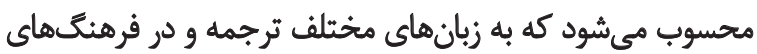

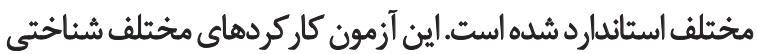

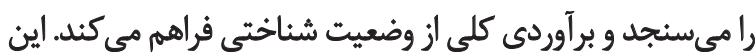

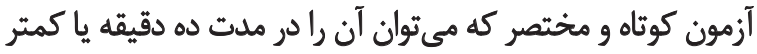

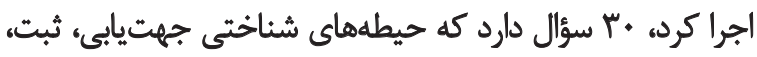

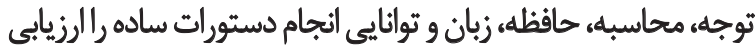

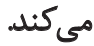

5. Beck depression inventory

6. Mini mental state examination
راكوتاهتر برآورد مي كنيند [ [ ] إين تجربه ذهنى در تحقيقات مختلف با رويكردها و روشهاى كوناكون مورد مطالعه قرار كرفته است. برخى مطالعات از يرسشنامههايى استفاده كردماند كه در آن آز آز

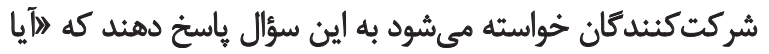

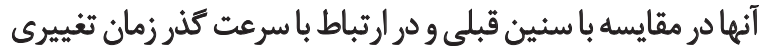

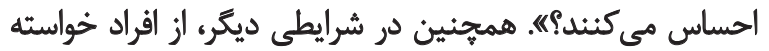

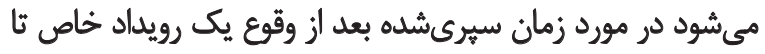
الحظه حاضر قضاوت كنئد.

يافته اصلى اين نوع يروهشها اين بوده است كه زمان فيزيكى با

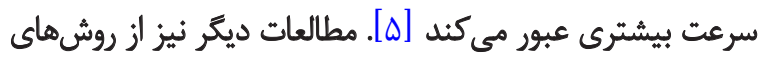

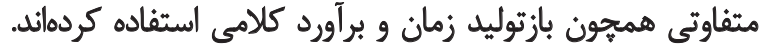

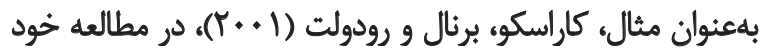

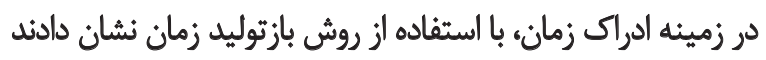

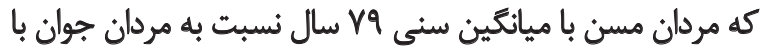

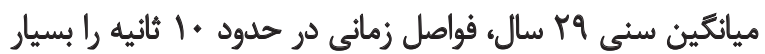

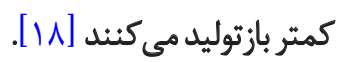

ازآنجايى كه تفاوتهاى فردى هرجند جزيى و كوخيك در ادراك

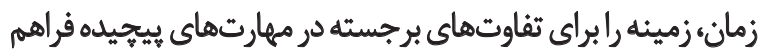

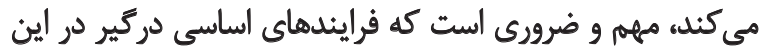

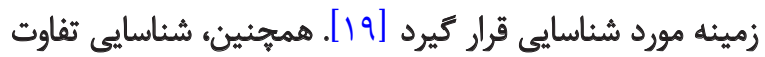

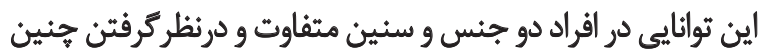

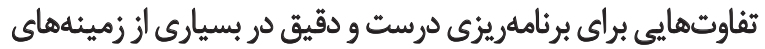

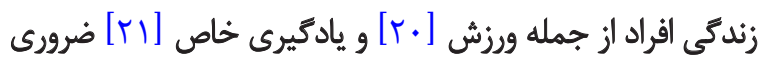

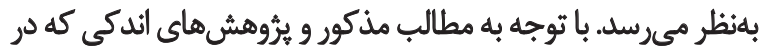

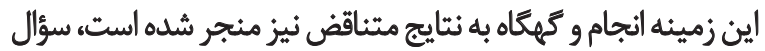

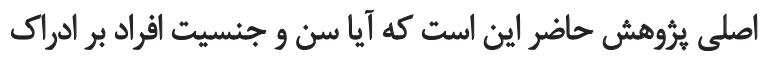
زمان ثأثير مى كذارد؟ حاض اين

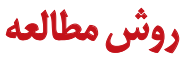

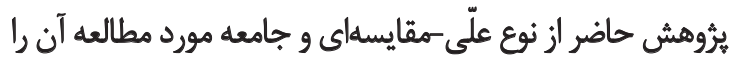

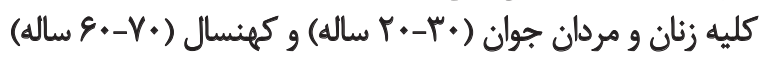

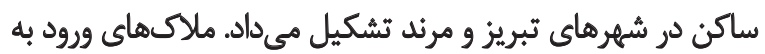

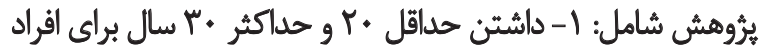

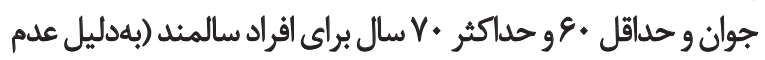

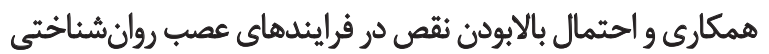

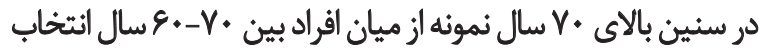

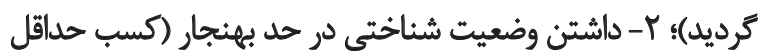

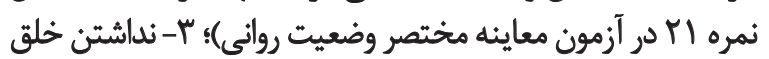

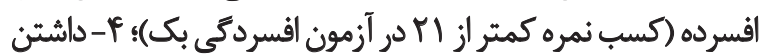

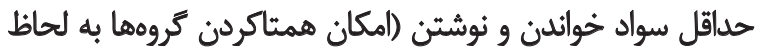

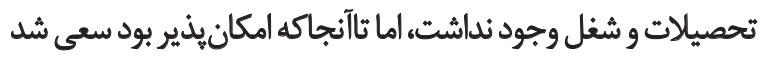

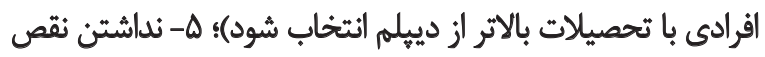




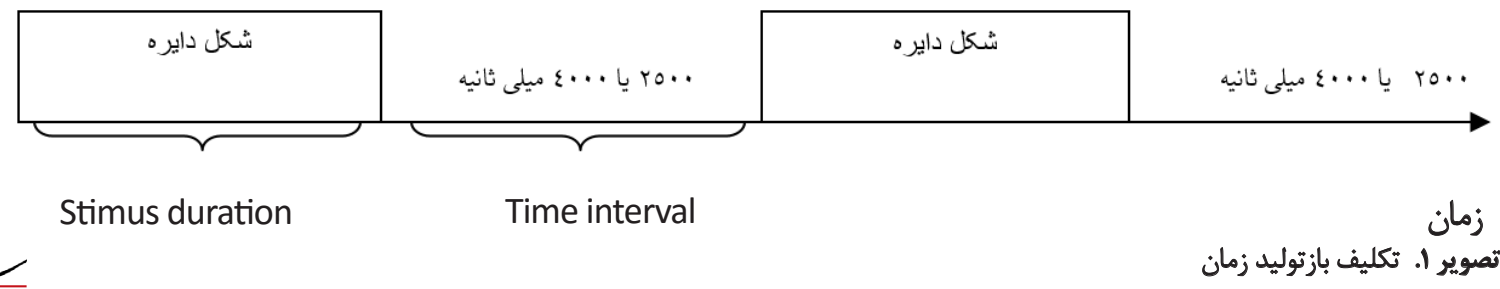

بود و بهصورت تصادفى ارائه مي شد. در اين مرحله، آزمودنى مي بايست

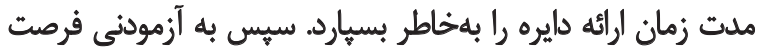

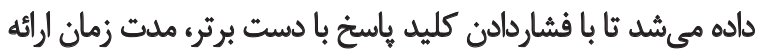

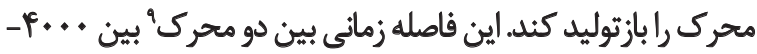

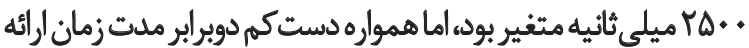

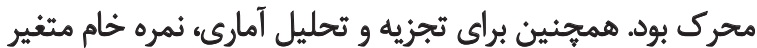

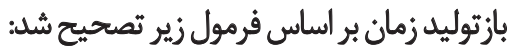

\section{Tstandard $/$ Tcorrected $=$ Treproduced - Tstandard}

در زَاره بالا، Tcorrected نمره زمان تصحيحشده متغير بازتوليد

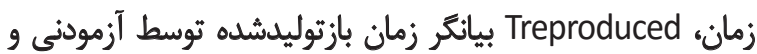
Tstandard

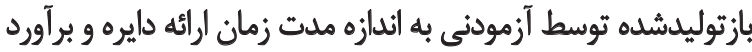

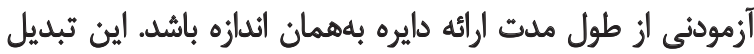

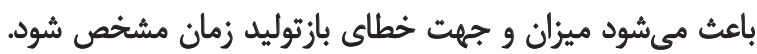

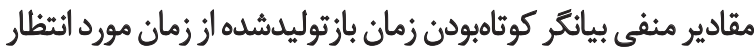

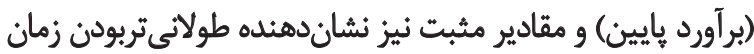

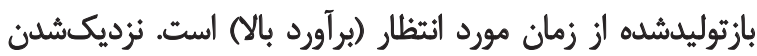

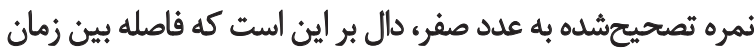

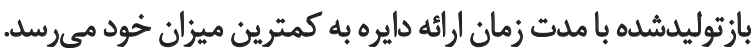
يس ازئ انتخاب افراد نمونه، تكليف مذكور براى هريك از آزمودنىها

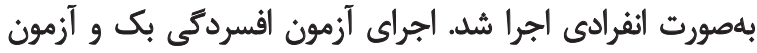

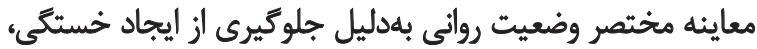

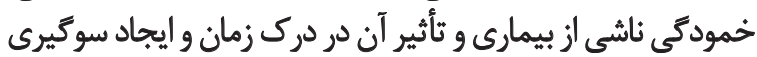

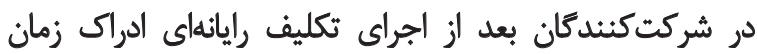

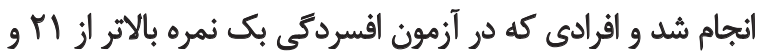

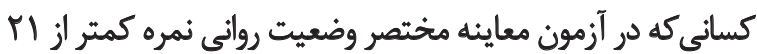

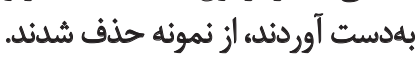

تكليف در محيطى آرام و درحالي كه فرد با حالت آرامش در مقابل

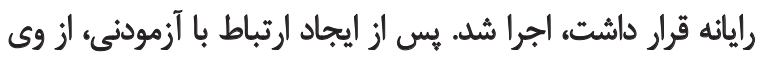

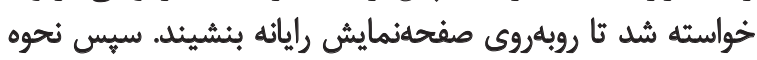

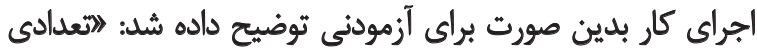

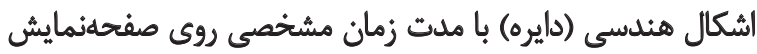

9. Time interval

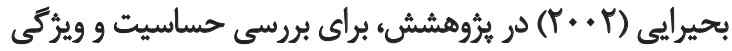

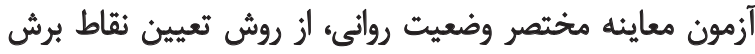

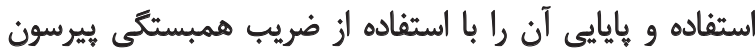

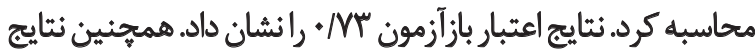

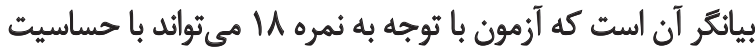

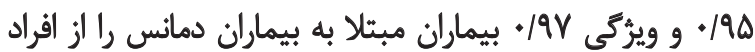

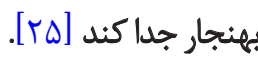

تكليف بازٔتوليد زمان

از ميان تكاليف اندازهيرى ادراك زمان، تكليف بازتوليد زمان

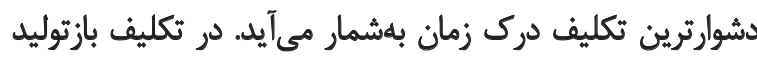

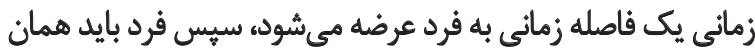

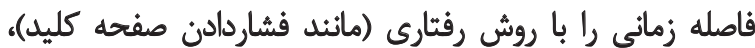

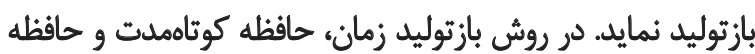

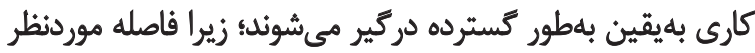

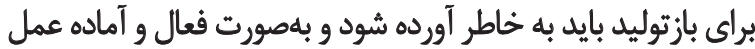

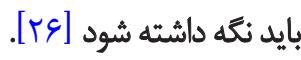

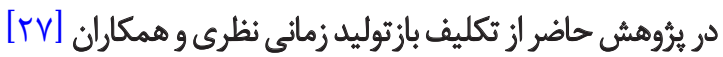

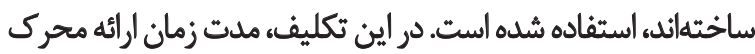

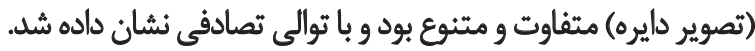

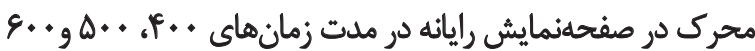

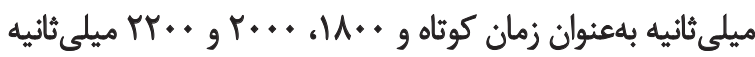

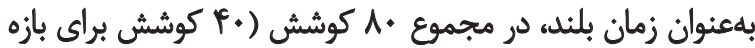

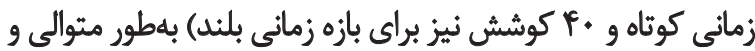

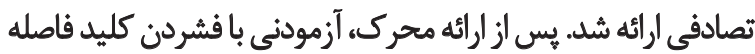

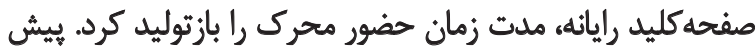

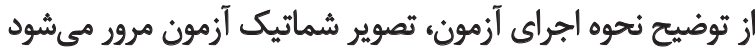

(تصوير شماره ()). ( نوضع نهواج)

همان طور كه شكل بالانشان مي دهد، شكل دايره يكبهيك ازئ طريق

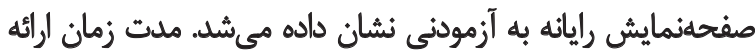

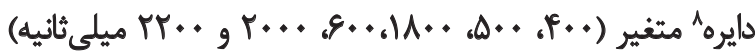

7. Time reproduction task

8. Stimulus duration 


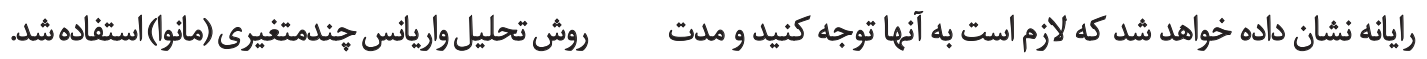

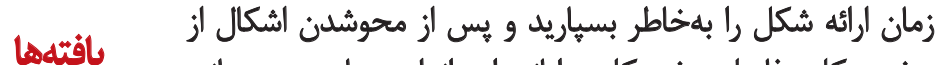

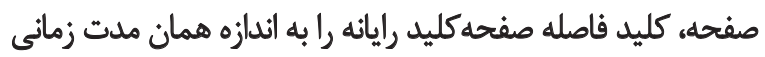
در بيروهش حاضر، تجزيه و تحليل آمارى دادههاى جمع آورى شاده كه بهخاطر سيردايد، فشار دهيد؟

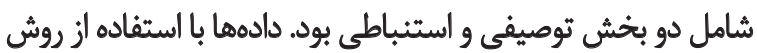

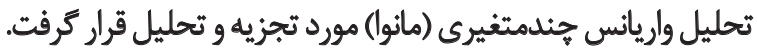

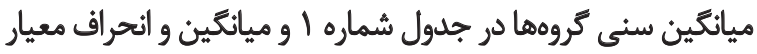

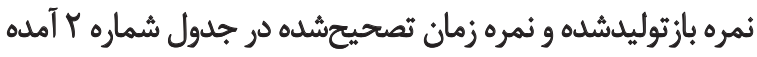

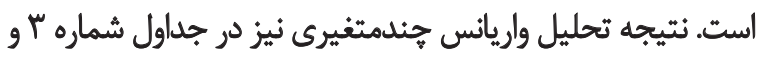
fi أشان داده شده است.

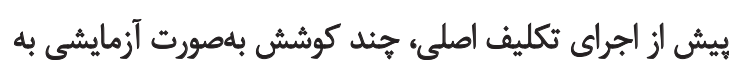

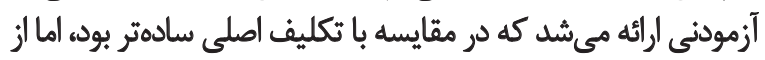

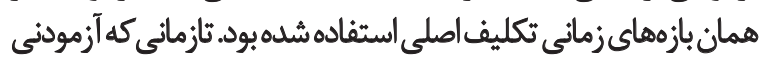

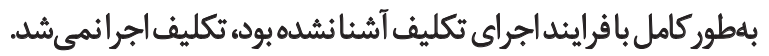

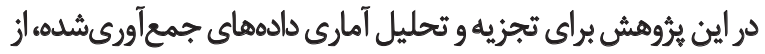

جدول ا. شاخصهاى ثوصيفى مربوط به سن آزمودنى ها در كروهها.

\begin{tabular}{|c|c|c|c|c|}
\hline انحراف معيار & مياتكين & تعداد & & \\
\hline * MIr & $r E / T$. & 10 & هرد & \multirow{2}{*}{ جوان } \\
\hline$\cdot|\Delta T|$ & $r \& / . V$ & 10 & ز & \\
\hline - NDA & $g \Delta / f^{e}$ & ir & هرد & \multirow[b]{2}{*}{ هسن } \\
\hline.$/ \Delta \Delta \&$ & $\varepsilon N / N$ & 10 & زن & \\
\hline
\end{tabular}

جدول r. ميانكين و انحراف معيار نمره بازتوليدشده و ئمره T تصحيحشده به تفكيك بازههاى زمانى و كروهها.

\begin{tabular}{|c|c|c|c|c|c|c|}
\hline مياتكين نمره T & انتحراف معيار & انحراف معيار نمره باز توليدشده & ميانكين نمره بازتوليدشده & سن & جنسيت & \\
\hline . MFtr & $. / \pi \| l$. & $1 \cdot f / T$ & $811 / r$ & جوان & & \multirow{6}{*}{ زمان كوتاه } \\
\hline$-.1+101$ & $\cdot r \cdot r q$ & $10 \cdot \pi$ & $p q 9 / 4$ & سالمند & هرد & \\
\hline - & - MVATE & Ire/f & $\Delta E / / \varepsilon$ & كل & & \\
\hline . $1 \cdot \Delta H M$ & .17199. & $r+\Delta / F$ & $\Delta W V$ & جوان & \multirow{3}{*}{ زن } & \\
\hline$-1+14$ & - Ragr & $19 r / \mathrm{V}$ & $p q 9 / 1$ & سالمند & & \\
\hline$-.+1+101$ & $\cdot / f+k r$ & $199 / 5$ & PANT & كل & & \\
\hline.$- /$ MVE & ./NTm & MATIS & Iffrt/ & جوان - & & \multirow{6}{*}{ زمان بلند } \\
\hline$-+/ F 194$ & - NFAFI & rav/I & $\| \varepsilon+/ V$ & سالمند & هرد & \\
\hline.$- / 4799$ & ./Neq. & หมู/ & $|m| v / 9$ & كل & & \\
\hline$-+/ N F T$ & . Irg.A & $r Y T /$ & "r/m/m & جوان & & \\
\hline.$- / 48.8$ & . / VAAmF & TAT/V & $1 \cdot V \Delta / V$ & سالمند & زن & \\
\hline.$- / 8 \cdot 18$ & $.119 .$. & $M T / M$ & $1194 / 0$ & كل & & \\
\hline
\end{tabular}

L

جدول ب. نتايج تحليل واريائس جندمتغيره در مورد تأثير سن و جنس بر ادراك زمان.

\begin{tabular}{|c|c|c|c|c|c|c|}
\hline مجذور اتا & سطع معنادارى & Df & قطعى f & الرخش & نام آزمون & الثي \\
\hline$\cdot / \Delta r$ & - Mpe & Ho. & 1/peqq &.$/ 91 Y$ & لامبداي ويلكز & جنسيث \\
\hline - / VVA & $+\cdot \cdot V$ & $r / \ldots$ & $\Delta / \Delta I V$ & - IATA & لامبداي ويلكز & سن \\
\hline
\end{tabular}


جدول F. نتايج تحليل واريائس جندمتغيرى براى مقايسه ميانكين تروهها در مدت زمان كوتاه و مدت زمان بلند.

\begin{tabular}{|c|c|c|c|c|c|c|}
\hline مجذور اتا & سطع معنادارى & $\mathrm{F}$ & درجات أزادى & مجذور مياتئين ها & مثغير وابسته & \\
\hline 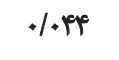 &.$/ N T$ & r/Mat & 1 & $+/ 194$ & زمان كوثاه & \multirow{2}{*}{ جنسيت } \\
\hline.$/+\mathrm{He}^{\mathrm{C}}$ &.$/ 118$ & I/MA & 1 & $+|+4|$ & زمان بلثد & \\
\hline .1 .89 & $. / \Delta)^{\circ}$ & ए/A१9 & 1 & . pees & زمان كوتاه & \multirow{2}{*}{ سن } \\
\hline.$/ 1 r^{r}$ &.$/ 0 r$ & $11 / \cdot v e$ & 1 & . Mre & زمان بلند & \\
\hline
\end{tabular}

L

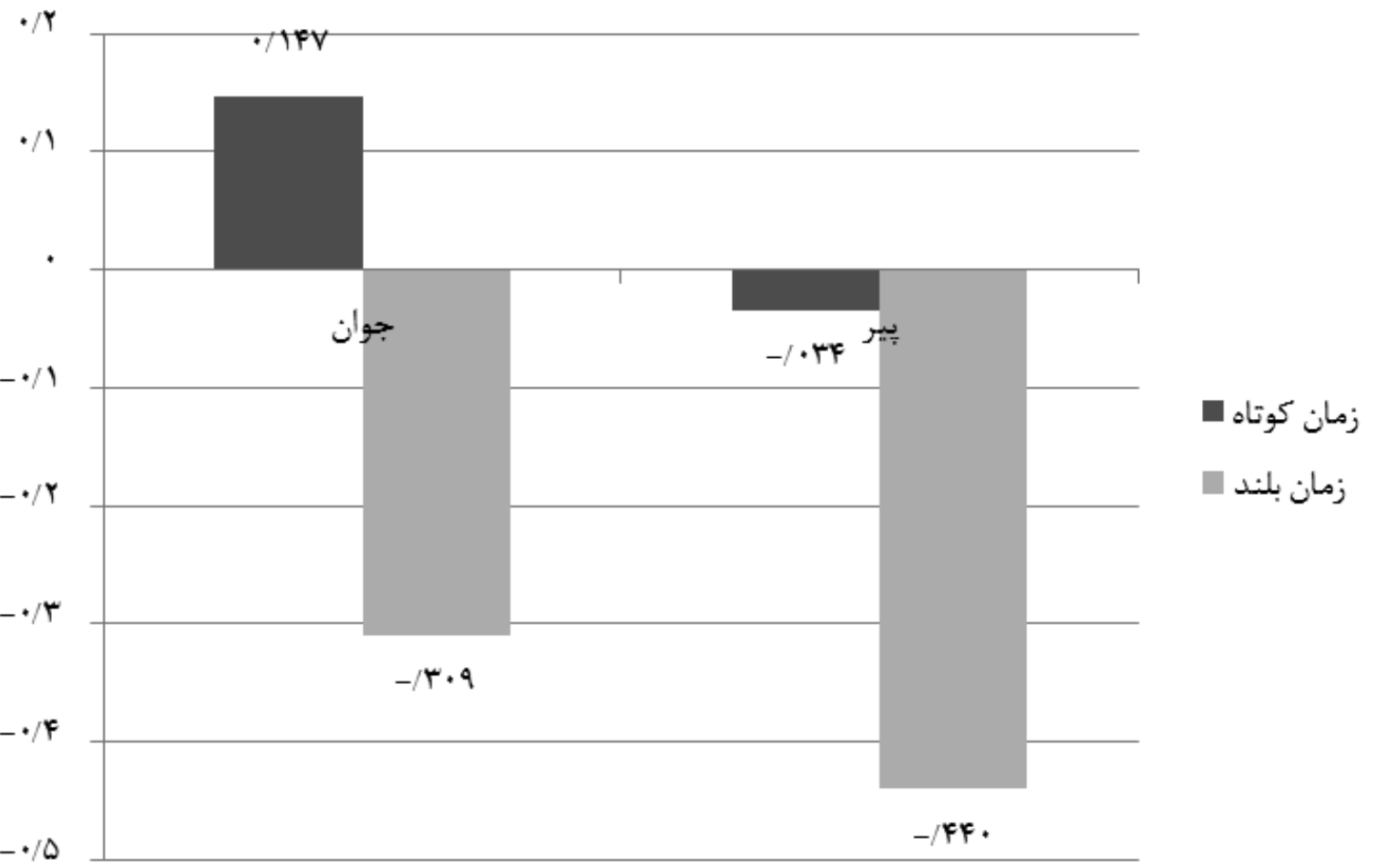

تصوير T. ميانكين نمره بازٔتوليد زمان (نمره T تصحيحشده) براى بازههاى زمانى كوتاه و بلئد به تفكيى كروه سئى جوان و سالمئد.

بين دو كروه (زن و مرد) در بازههاى زمانى كوثاه و بلند تثفاوت

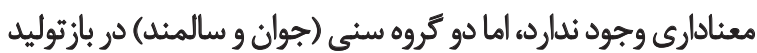

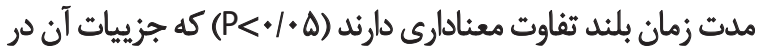
تصوير شماره Y قابل مشاهده است.

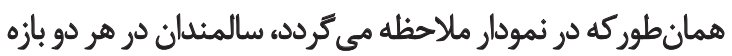

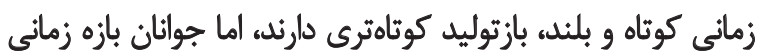

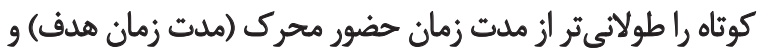

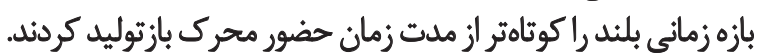

بحث

هدف اصلى اين مطالعه، باسخ به اين سؤال بود كه هآيا سن و

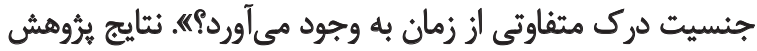

بررسى مفروضههاى اصلى تحليل واريانس جندمتغيرى نشان داد

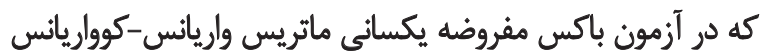

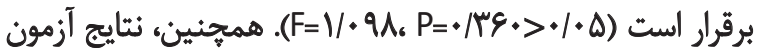

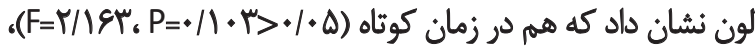

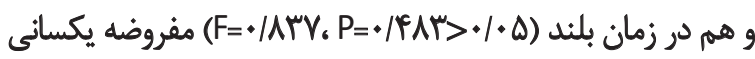
واريانس ها برقرار است. نتايج مقايسه تحليل واريانس جندمثغيرى (MANOVA) در

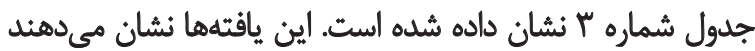

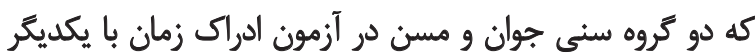

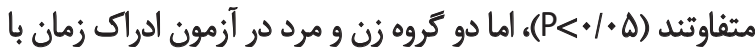

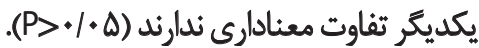
براساس جدول شماره f، مشاهده مىشود كه در متغير جنسيت 


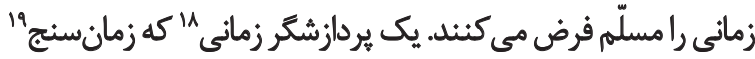

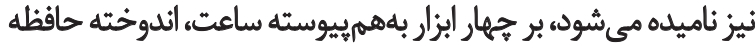

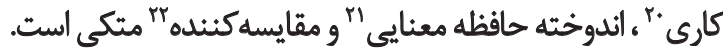

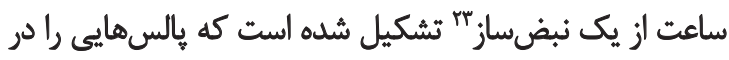

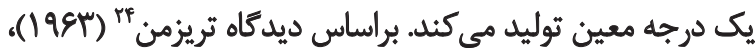

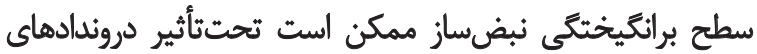

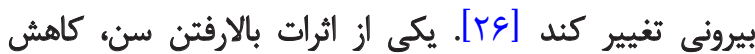

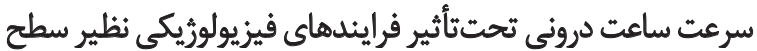

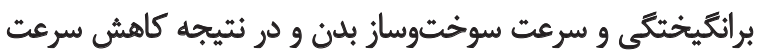

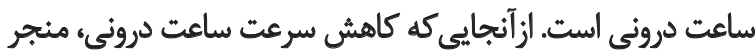

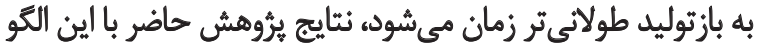

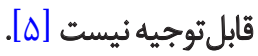

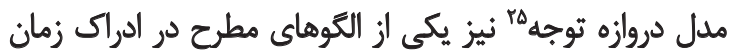

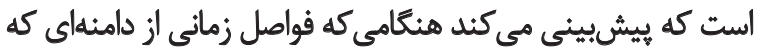

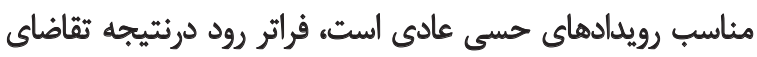

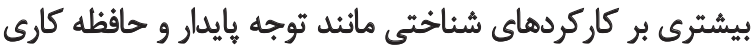

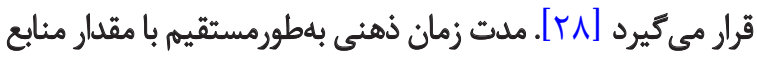

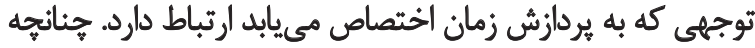

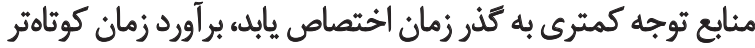

خواهد بود [هاب.

براساس تغييرات شناختى مربوط به كهنسالى، افزايش سن بر برائر

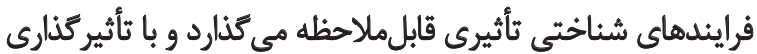

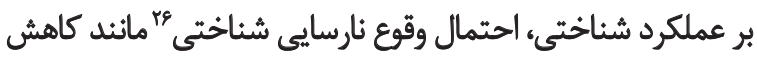

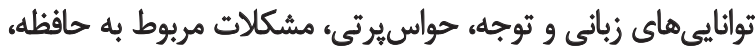

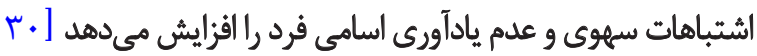

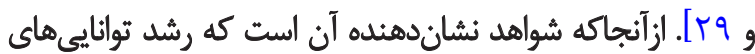

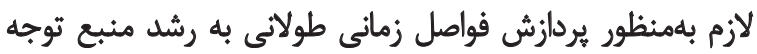

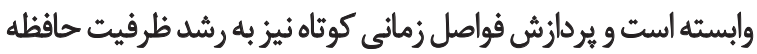

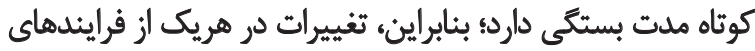

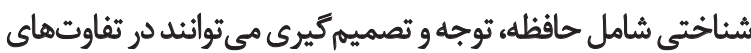

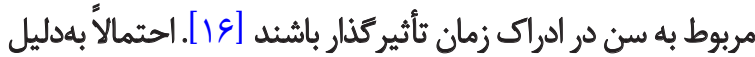

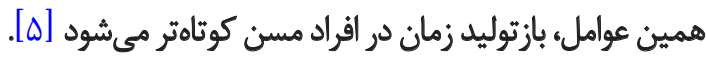

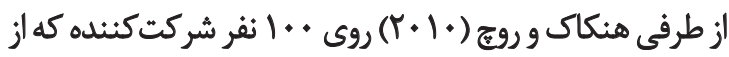

18. Temporal processor

19. Timer

20. Working memory store

21. Reference memory store

22. Comparator

23. Pacemaker

24. Treisman

25. attentional gate model

26. cognitive failure

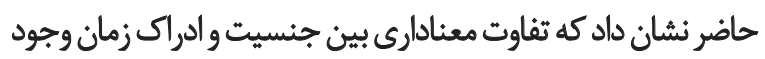

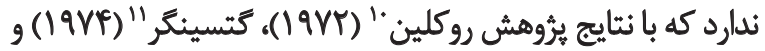

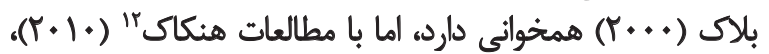

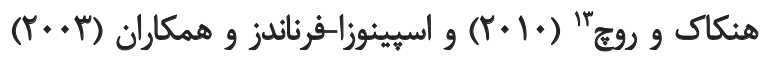

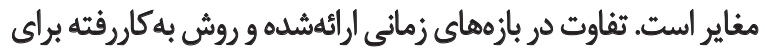

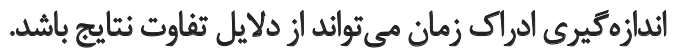

برخى يروهشكران از جمله بلاك، هنكاى و زكاى"

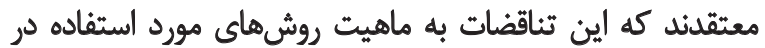

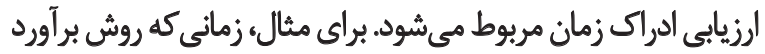

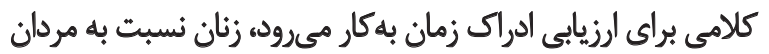

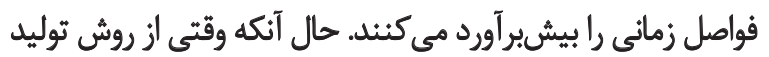

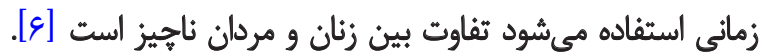

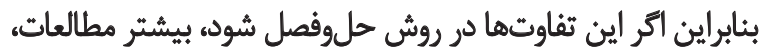
الكوى همسانى را نشان خواهند داد [ [ ]

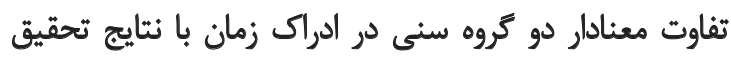

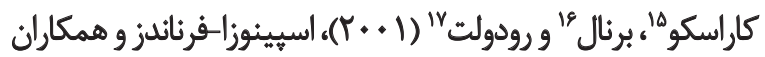

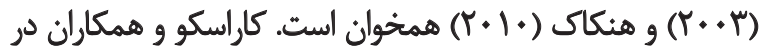

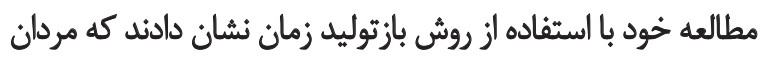

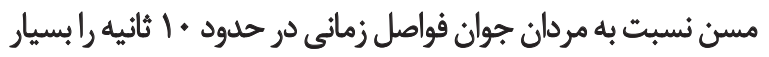

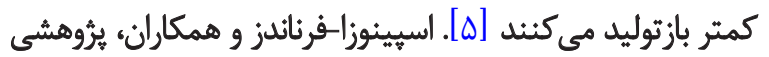

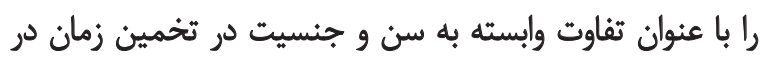

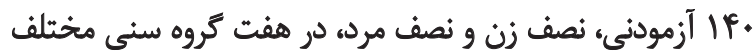

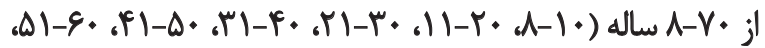

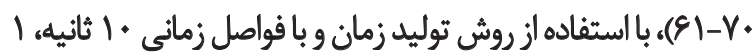

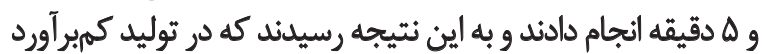

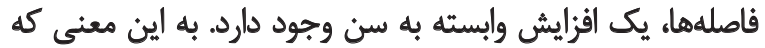

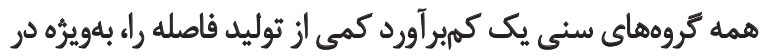

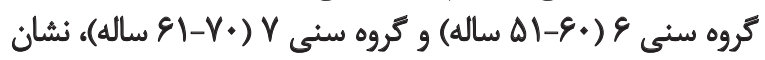
دادند [ه]. - ان.

در اين راستا الكو و نظريههاى مختلفى مطرح شده است كه نتايج

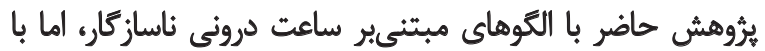

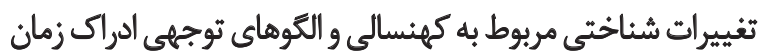

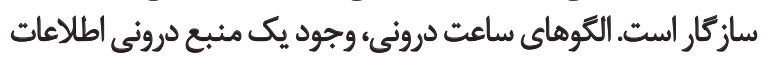

10. Roeckelien

11. Getsinger

12. Hancock

13. Rausch

14. Zakay

15. Carrasco

16. Bernal

17. Redolat 
تجربى بيشتر نياز دارد.

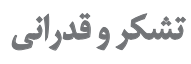

دريايان، از افراد جوان و سالمندى كه در اين يُروهش شركت كردند، صميمانه قدردانى مى تردد.

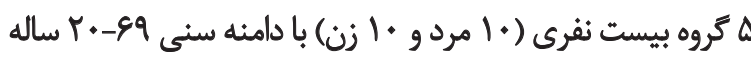

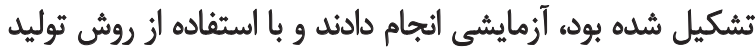

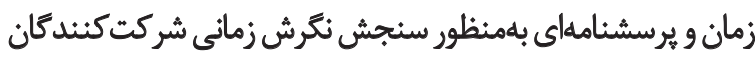

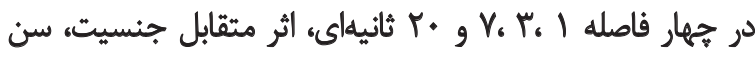

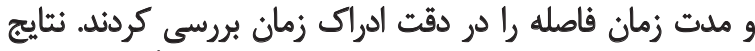

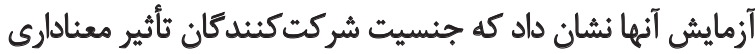

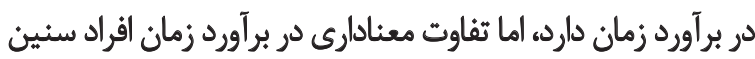

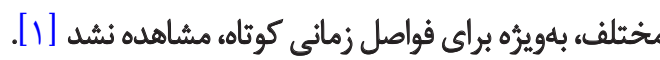

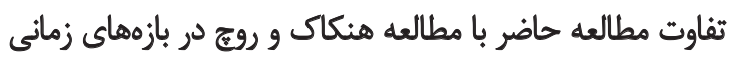

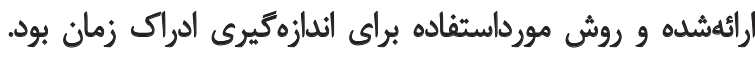

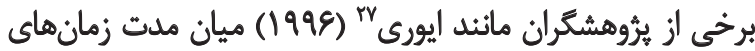

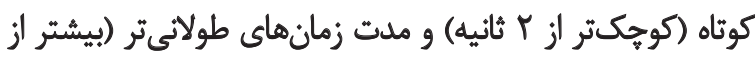

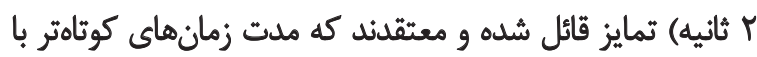

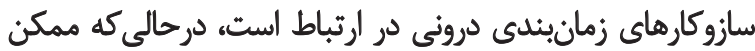

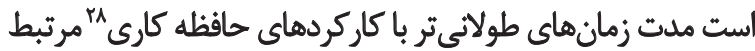

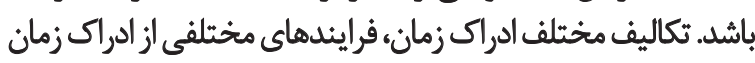

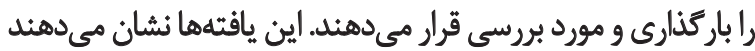

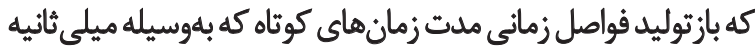

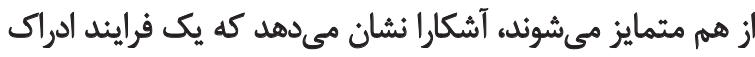

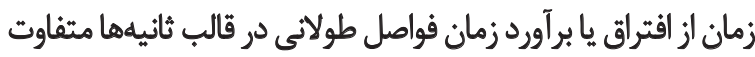

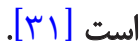

\section{نتيجه تيرى نهايى}

يافتهاى اين بثروهش نشان مى دهد كه سن بر ادراك زمان زمان تأثير

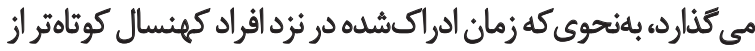

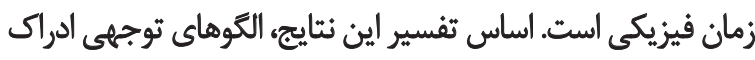

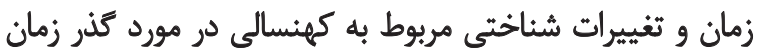

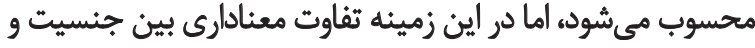

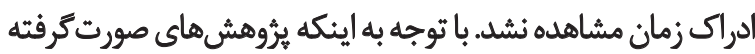

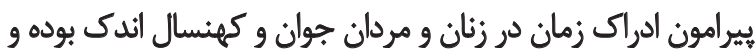

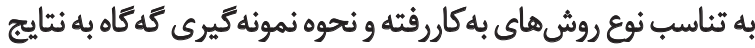

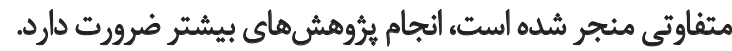

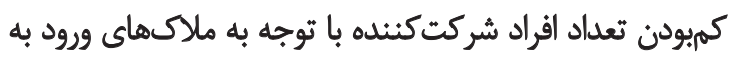

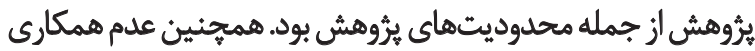

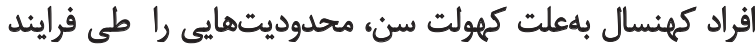

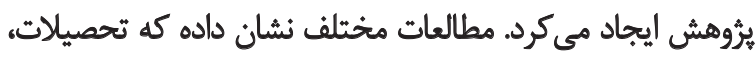

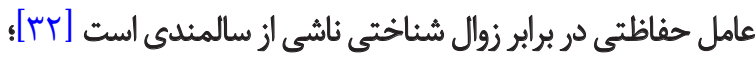

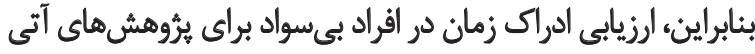

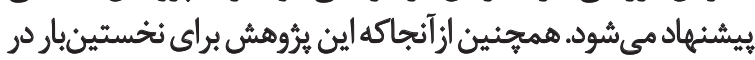

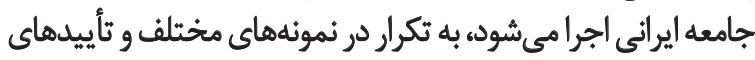




\section{References}

[1] Hancock PA, Rausch R. The effects of sex, age, and interval duration on the perception of time. Acta Psychologica. 2010; 133(2):170-9.

[2] Toplak ME, Rucklidge JJ, Hetherington R, John SC, Tannock $\mathrm{R}$. Time perception deficits in attention deficit/hyperactivity disorder and comorbid reading difficulties in child and adolescent samples. Journal of Child Psychology and Psychiatry. 2003; 44(6):888-903.

[3] Church RM. A concise introduction to the scalar timing theory. In: Meck WH, editor. Functional and neural mechanisms of interval timing. Boca Raton: CRC Press; 2003, pp. 3-32.

[4] Grondin S. From physical time to the first and second moments of psychological time. Psychological Bulletin. 2001; 127(1):22-44.

[5] Espinosa-Fernández L, Miró E, Cano M, Buela-Casal G. Agerelated changes and gender differences in time estimation. Acta Psychologica. 2003; 112(3):221-32.

[6] Block RA, Hancock PA, Zakay D. Sex differences in duration judgments: A meta-analytic review. Memory \& Cognition. 2000; 28(8):1333-46.

[7] Hancock PA. The effect of age and sex on the perception of time in life. American Journal of Psychology. 2010; 123(1):1-3.

[8] Maitland SB, Intrieri RC, Schaie WK, Willis SL. Gender differences and changes in cognitive abilities across the adult life span. Aging, Neuropsychology, and Cognition. 2000; 7(1):32-53.

[9] Jorm AF, Anstey KJ, Christensen H, Rodgers B. Gender differences in cognitive abilities: The mediating role of health state and health habits. Intelligence. 2004; 32(1):7-23

[10] Gur RC, Mozley LH, Mozley PD, Resnick SM, Karp JS, Alavi $\mathrm{A}$, et al. Sex differences in regional cerebral glucose metabolism during a resting state. Science. 1995; 267(5197):528-31.

[11] Hancock PA. Body temperature influence on time perception. Journal of Ggeneral Psychology. 1993; 120(3):197-216.

[12] Block RA, Hancock PA, Zakay D. Sex differences in duration judgments: A meta-analytic review. Memory \& Cognition. 2000; 28(8):1333-46.

[13] Berk L. Development through the lifespan] [S. Mohammadi, Persian trans]. Tehran: Arasbaran; 2007.

[14] Casini L, Ivry RB. Effects of divided attention on temporal processing in patients with lesions of the cerebellum or frontal lobe. Neuropsychology. 1999; 13(1):10-21.

[15] Chudasama Y, Robbins TW. Functions of frontostriatal systems in cognition: Comparative neuropsychopharmacological studies in rats, monkeys and humans. Biological Psychology. 2006; 73(1):19-38

[16] Zélanti PS, Droit-Volet S. Cognitive abilities explaining agerelated changes in time perception of short and long durations. Journal of Experimental Child Psychology. 2011; 109(2):143-57.

[17] Poppel E. Lost in time: A historical frame, elementary processing units and the 3-second window. Acta Neurobiologiae Experimentalis. 2004; 64(3):295-302.
[18] Carrasco MC, Bernal MC, Redolat R. Time estimation and aging: A comparison between young and elderly adults. International Journal of Aging and Human Development. 2001; 52(2):91-101

[19] Rammsayer TH, Rammstedt B. Sex-related differences in time estimation: The role of personality. Personality and Individual Differences. 2000; 29(2):301-12.

[20] Wittmann M, Paulus MP. Intertemporal choice: Neuronal and psychological determinants of economic decisions. Journal of Neuroscience, Psychology, and Economics. 2009; 2(2):71-74.

[21] Grondin S, Dionne G, Malenfant N, Plourde M, Cloutier ME Jean C. Temporal processing skills of children with and without specific language impairment. Canadian Journal of Speech-Language Pathology and Audiology. 2007; 31(1):38-46.

[22] Gil S, Droit-Volet S. Time perception, depression and sadness. Behavioural Processes. 2009; 80(2):169-76.

[23] Pouthas V, Perbal S. Time perception depends on accurate clock mechanisms as well as unimpaired attention and memory processes. Acta Neurobiologiae Experimentalis. 2004 64(3):367-86

[24] Besharat MA. [The relationship between alexithymia anxiety, depression, psychological distress and psychological well-being (Persian)] [Scientific research]. Tabriz: University of Tabriz; 2008.

[25] Bohiraie AR. [An elementary camparision of efficacy minimental state examination (Persian)]. In: Taaghol AS, editor. Proceedings of Aging Issues in Iran and Other Countries. Tehran: Ketab Ashna; 2002

[26] Ekhtiari H, Jannati AS, Parhizgar B, Mokri A. [Time perception and evaluation methods: A pilot study to test the Persian (Persian)]. Journal of Cognitive Science. 2003; 5:36-49.

[27] Nazari MA, Mirloo MM, Asadzadeh S. [Time perception error in processing emotional Persian words (Persian)]. Advances in Cognitive Science. 2011; 13(1):37-48.

[28] Toplak ME, Dockstader C, Tannock R. Temporal information processing in ADHD: Findings to date and new methods. Journal of Neuroscience Methods. 2006; 151(1):15-29.

[29] Dixon R, Backman L, Nilsson LG. New frontiers in cognitive aging. Oxford: Oxford University Press; 2004.

[30] Wallace JC. Confirmatory factor analysis of the cognitive failures questionnaire: Evidence for dimensionality and construct validity. Personality and Individual Differences. 2004; 37(2):307-24.

[31] Smith A, Tyler E, Rogeres JW, Newman S, Rubia K. Evidence for a pure time perception deficit in children with ADHD. Journal of Child Psychology and Psychiatry. 2002; 43(4):529-542.

[32] Nejati V. Cognitive-executive functions of brain frontal lobe in aged adults. Journal of Behavioral Sciences. 2010; 4(1):17-18 